\title{
On-Top Pair-Density Interpretation of Spin Density Functional Theory, with Applications to Magnetism
}

\author{
JOHN P. PERDEW, MATTHIAS ERNZERHOF, AND KIERON BURKE \\ Department of Physics and Quantum Theory Group, Tulane University, New Orleans, Louisiana \\ 70118
}

\author{
ANDREAS SAVIN* \\ Laboratoire Dynamique des Interactions Moléculaires, Université Pierre et Marie Curie, F-75252 \\ Paris, France
}

Received August 30, 1995; accepted October 23, 1995

\begin{abstract}
The on-top pair density $P(\mathbf{r}, \mathbf{r})$ gives the probability that one electron will be found on top of another at position $\mathbf{r}$. We find that the local spin density (LSD) and generalized gradient (GGA) approximations for exchange and correlation predict this quantity with remarkable accuracy. We show how this fact and the usual sum-rule arguments explain the success of these approximations for real atoms, molecules, and solids, where the electron spin densities do not vary slowly over space. Self-consistent LSD or GGA calculations make realistic predictions for the total energy $E$, the total density $n(\mathbf{r})$, and the on-top pair density $P(\mathbf{r}, \mathbf{r})$, even in those strongly "abnormal" systems (such as stretched $\mathrm{H}_{2}$ ) where these approximations break symmetries and yield unrealistic spin magnetization densities $m(\mathbf{r})$. We then suggest that ground-state ferromagnetic iron is a "normal" system, for which for LSD or GGA $m(\mathbf{r})$ and the related local spin moment are trustworthy, but that iron above the Curie temperature and antiferromagnetic clusters at all temperatures are abnormal system for which the on-top pair density interpretation is more viable than the standard physical interpretation. As an example of a weakly abnormal system, we consider the four-electron ion with nuclear charge $Z \rightarrow \infty$. (c) 1997 John Wiley \& Sons, Inc.
\end{abstract}

\footnotetext{
* Present Address: Laboratoire de Chimie Theoretique, Université Pierre et Marie Curie, F-75252 Paris, France. 


\section{Introduction}

The spin density functional theory of Kohn and Sham [1-4] is a formally exact prescription for the ground-state electron spin-densities $n_{\uparrow}(\mathbf{r})$ and $n_{\downarrow}(\mathbf{r})$ and the total energy $E$ of a many-electron system, via the solution of a self-consistent oneelectron Schrödinger equation. When the imperfectly known exchange-correlation energy functional $E_{x c}\left[n_{\uparrow}(\mathbf{r}), n_{\downarrow}(\mathbf{r})\right]$ is treated in the local spin density [1-4]

$$
E_{x c}^{\mathrm{LSD}}\left[n_{\uparrow}, n_{\downarrow}\right]=\int d^{3} r n(\mathbf{r}) \epsilon_{x c}\left(n_{\uparrow}(\mathbf{r}), n_{\downarrow}(\mathbf{r})\right)
$$

or generalized gradient approximations $[5,6]$

$$
\begin{aligned}
E_{x c}^{\mathrm{GGA}} & {\left[n_{\uparrow}, n_{\downarrow}\right] } \\
& =\int d^{3} r f\left(n_{\uparrow}(\mathbf{r}), n_{\downarrow}(\mathbf{r}), \nabla n_{\uparrow}(\mathbf{r}), \nabla n_{\downarrow}(\mathbf{r})\right),
\end{aligned}
$$

first-principles calculations of useful accuracy can be made for atoms, molecules, and solids.

Approximations (1) and (2) are valid when the spin densities vary slowly over space, on the scale of the diameter of the exchange-correlation hole surrounding an electron. This condition is never satisfied in real systems, but the approximations work anyway. In the absence of an external magnetic field, only the total density $n(\mathbf{r})=n_{\uparrow}(\mathbf{r})+$ $n_{\downarrow}(\mathbf{r})$ is formally needed, but, in practice, one still needs spin densities to get accurate energies for open-shell systems [7]. In systems that we shall call "normal," these approximations deliver realistic results for the total density $n(\mathbf{r})=n_{\uparrow}(\mathbf{r})+$ $n_{\downarrow}(\mathbf{r})$, the spin magnetization $m(\mathbf{r})=n_{\uparrow}(\mathbf{r})-$ $n_{\downarrow}(\mathbf{r})$, and the total energy $E$. In strongly "abnormal" systems, such as the $\mathrm{Cr}_{2}$ molecule [8], the predicted $m(\mathbf{r})$ is quite incorrect but $n(\mathbf{r})$ and $E$ are still usefully accurate.

It is a fact that the approximations work as described above, in extensive tests for real systems. The question is, Why? Previous justifications of LSD or GGA [9-13], although illuminating, have not fully explained the role of the local spin densities, the relevance of the uniform electron gas [which provides the input to Eq. (1)], or the distinction between normal and abnormal systems.

In our attempt [14-18] to answer this question, we found it useful to think about the on-top electron pair density $P(\mathbf{r}, \mathbf{r})$, as defined in the following section. In the second to fourth sections, we argue that, in the absence of an external magnetic field $B(\mathbf{r})$, LSD and GGA typically deliver accurate values for $P(\mathbf{r}, \mathbf{r})$ and for the closely related energy $E$, even when they do not predict realistic results for $m(\mathbf{r})$. Moreover, when $m(\mathbf{r})$ goes wrong, it does so in order to make $P(\mathbf{r}, \mathbf{r})$ right.

LSD and GGA provide our most realistic firstprinciples descriptions of ferromagnetic solids like iron [19-21] and of antiferromagnetic solids like iron oxide [22] or chromium [23]. But can we trust the predicted $m(\mathbf{r})$ for these systems? Why does the LSD prediction of $m(\mathbf{r})$ for Fe vanish [24] at a temperature much higher than the measured Curie temperature? Should we believe the predicted spin moment on an atom in a magnetic solid? In the fifth section, we address these questions within the on-top pair-density interpretation [15] of spin density functional theory. We use atomic units $\left(e^{2}=\hbar\right.$ $=m=1$ ) exclusively.

\section{Pair Density and Exchange-Correlation Hole}

From an $N$-electron wave function $\Psi$, we may construct the spin density

$$
\begin{aligned}
n_{\sigma}(\mathbf{r})= & N \sum_{\sigma_{2}, \ldots, \sigma_{N}} \int d^{3} r_{2} \ldots d^{3} r_{N} \\
& \times\left|\Psi\left(\mathbf{r}, \sigma, \mathbf{r}_{2}, \sigma_{2}, \ldots, \mathbf{r}_{N}, \sigma_{N}\right)\right|^{2}
\end{aligned}
$$

and the pair density

$$
\begin{aligned}
P\left(\mathbf{r}, \mathbf{r}^{\prime}\right)= & N(N-1) \sum_{\sigma_{1}, \ldots, \sigma_{N}} \int d^{3} r_{3} \ldots d^{3} r_{N} \\
& \times\left|\Psi\left(\mathbf{r}, \sigma_{1}, \mathbf{r}^{\prime}, \sigma_{2}, \ldots, \mathbf{r}_{N}, \sigma_{N}\right)\right|^{2} .
\end{aligned}
$$

While $n_{\sigma}(\mathbf{r}) d^{3} r$ is the probability of finding an electron with spin $\sigma$ in the volume element $d^{3} r$, $P\left(\mathbf{r}, \mathbf{r}^{\prime}\right) d^{3} r d^{3} r^{\prime}$ is the probability of finding a pair of electrons, one in $d^{3} r$ and another in $d^{3} r^{\prime}$. The expectation value of a spin-dependent external potential $v_{\sigma}(\mathbf{r})$ is

$$
\int d^{3} r n_{\sigma}(\mathbf{r}) v_{\sigma}(\mathbf{r})
$$

while that of the electron-electron repulsion is

$$
\frac{1}{2} \int d^{3} r d^{3} r^{\prime} \frac{P\left(\mathbf{r}, \mathbf{r}^{\prime}\right)}{\left|\mathbf{r}-\mathbf{r}^{\prime}\right|}
$$


The on-top pair density $P(\mathbf{r}, \mathbf{r})$ gives the probability of finding one electron on top of another at the position $\mathbf{r}$.

In Kohn-Sham theory [1-4], we use a coupling constant $\lambda[9,25]$ to connect the ground-state wave function $\left(\Psi_{\lambda=1}\right)$ of the real system to that of a noninteracting system $\left(\Psi_{\lambda=0}\right)$ with the same spin densities. Writing the electron-electron interaction as $\lambda /\left|\mathbf{r}^{\prime}-\mathbf{r}\right|$, we adjust the external potential $v_{\sigma}^{\lambda}(\mathbf{r})$ until the ground-state wave function $\Psi_{\lambda}$ has the physical $(\lambda=1)$ spin densities $n_{\uparrow}(\mathbf{r})$ and $n_{\downarrow}(\mathbf{r}) . \Psi_{\lambda}$ is just that wave function which yields the spin densities $n_{\uparrow}(\mathbf{r}), n_{\downarrow}(\mathbf{r})$, and minimizes the expectation value of $\hat{T}+\lambda \hat{V}_{e e}$ [26], where $\hat{T}$ and $\hat{V}_{e e}$ are the kinetic and electron-electron repulsion energy operators.

We define the exchange-correlation hole density $n_{x c, \lambda}\left(\mathbf{r}, \mathbf{r}^{\prime}\right)$ at $\mathbf{r}^{\prime}$ around an electron at $\mathbf{r}$ via

$$
P_{\lambda}\left(\mathbf{r}, \mathbf{r}^{\prime}\right)=n(\mathbf{r})\left[n\left(\mathbf{r}^{\prime}\right)+n_{x c, \lambda}\left(\mathbf{r}, \mathbf{r}^{\prime}\right)\right]
$$

and the exchange-correlation energy as

$$
\begin{aligned}
E_{x c}\left[n_{\uparrow}(\mathbf{r}), n_{\downarrow}(\mathbf{r})\right] & \\
= & \int_{0}^{1} d \lambda \frac{1}{2} \int d^{3} r \int d^{3} r^{\prime} \frac{n(\mathbf{r}) n_{x c, \lambda}\left(\mathbf{r}, \mathbf{r}^{\prime}\right)}{\left|\mathbf{r}^{\prime}-\mathbf{r}\right|} \\
& =N \int_{0}^{1} d \lambda \int_{0}^{\infty} d u 2 \pi u\left\langle n_{x c, \lambda}(u)\right\rangle,
\end{aligned}
$$

where

$$
\left\langle n_{x c, \lambda}(u)\right\rangle=\int \frac{d \Omega_{\mathbf{u}}}{4 \pi} \frac{1}{N} \int d^{3} r n(\mathbf{r}) n_{x c, \lambda}(\mathbf{r}, \mathbf{r}+\mathbf{u})
$$

is the system- and spherically averaged exchange -correlation hole density at separation $u=\left|\mathbf{r}^{\prime}-\mathbf{r}\right|$ and $N=\int d^{3} r n(\mathbf{r})$ is the electron number. The exchange hole $n_{x}=n_{x c, \lambda=0}$ generates the exchange energy, which normally for most practical purposes is that of Hartree-Fock theory.

The LSD approximation [1-4] is

$$
n_{x c, \lambda}^{\mathrm{LSD}}(\mathbf{r}, \mathbf{r}+\mathbf{u})=n_{x c, \lambda}^{u n i f}\left(n_{\uparrow}(\mathbf{r}), n_{\downarrow}(\mathbf{r}) ; u\right),
$$

where $n_{x c, \lambda}^{u n i f}\left(n_{\uparrow}(\mathbf{r}), n_{\downarrow}(\mathbf{r}) ; u\right)$ is the hole density for an electron gas with uniform spin densities $n_{\uparrow}(\mathbf{r}), n_{\downarrow}(\mathbf{r})$ and coupling constant $\lambda$. The Perdew-Wang 1991 GGA approximation $[5,6]$ is more complicated, but has the same value and electron -electron cusp as Eq. (10) in the limit $u \rightarrow 0$.

Strong arguments have been given $[9,10]$ for the accuracy of LSD or GGA in real systems. For example, these approximations respect the sum rule

$$
\int_{0}^{\infty} d u 4 \pi u^{2}\left\langle n_{x c, \lambda}(u)\right\rangle=-1 .
$$

But these arguments provide no link between the hole density and the local spin densities, except in the limit of slowly varying densities.

Ziegler et al. [11] showed that, when the Kohn-Sham $(\lambda=0)$ wave function is a single Slater determinant, LSD is exact for the on-top $(u=0)$ exchange hole:

$$
\begin{aligned}
n_{x}(\mathbf{r}, \mathbf{r}) & =n_{x}^{\text {unif }}\left(n_{\uparrow}(\mathbf{r}), n_{\downarrow}(\mathbf{r}) ; u=0\right) \\
& =-\left[1+\zeta^{2}(\mathbf{r})\right] n(\mathbf{r}) / 2,
\end{aligned}
$$

where $\zeta(\mathbf{r})=\left(n_{\uparrow}(\mathbf{r})-n_{\downarrow}(\mathbf{r})\right) / n(\mathbf{r})$ is the relative spin polarization. Harris [12] pointed out that, in the fully polarized $(|\zeta| \rightarrow 1)$ or strongly interacting $(\lambda \rightarrow \infty)$ limits, $n_{x c, \lambda}^{\mathrm{LSD}}(\mathbf{r}, \mathbf{r})=-n(\mathbf{r})$ is also exact. It had been suspected $[25,27]$ that $n_{x c, \lambda}^{\text {LSD }}(\mathbf{r}, \mathbf{r})$ was also exact more generally, at least for metal surfaces, but this possibility is now disproved [14].

Nevertheless, we recently found [14-17] that LSD gives a remarkably accurate account of the system-averaged on-top hole density at full coupling strength, $\left\langle n_{x c, \lambda=1}(u=0)\right\rangle$ or equivalently of the volume integral of the on-top pair density, $\int d^{3} r P_{\lambda=1}(\mathbf{r}, \mathbf{r})$. Figure 1 shows the "universal curve $^{\prime \prime}$ for spin-unpolarized $(\zeta=0)$ systems, $\left\langle n_{x c, \lambda=1}(u=0)\right\rangle /\langle n\rangle$ vs. $\left\langle r_{s}\right\rangle$, where

$$
r_{s}=(3 / 4 \pi n)^{1 / 3}
$$

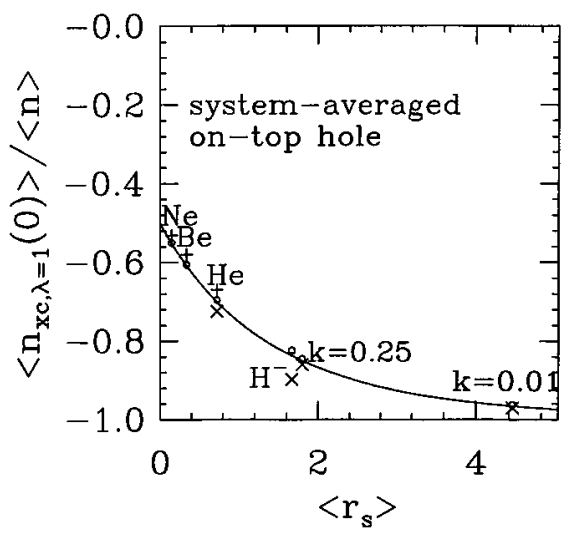

FIGURE 1. Universal curve for the system-averaged on-top hole density in spin-unpolarized systems. The solid curve is for the uniform electron gas [Eqs. (15) and (16)]. The circles, crosses, and plus signs are explained in the text. 
and

$$
\begin{gathered}
\langle n\rangle=\int d^{3} r n^{2}(\mathbf{r}) / \int d^{3} r n(\mathbf{r}), \\
\left\langle r_{s}\right\rangle=\int d^{3} r n^{2}(\mathbf{r}) r_{s}(\mathbf{r}) / \int d^{3} r n^{2}(\mathbf{r}) .
\end{gathered}
$$

The crosses in Figure 1 represent either almostexact numerical calculations for real atoms or exact analytic results for Hooke's atom [28] (two electrons bound to a center by a spring of force constant $k$ ). The pluses represent configuration interaction (CI) results [17], which underestimate the depth of the on-top hole because of the difficulty in representing the electron-electron cusp in a finite one-particle basis set. The circles indicate LSD values (evaluated using the exact or almostexact spin densities), and the solid curve is for the uniform electron gas:

$$
n_{x c, \lambda=1}^{u n i f}(n / 2, n / 2 ; u=0)=n\left[g^{\text {unif }}\left(r_{s} ; 0\right)-1\right],
$$

where [17]

$$
g^{u n i f}\left(r_{s} ; 0\right) \approx D\left[\left(\gamma+r_{s}\right)^{3 / 2}+\beta\right] e^{-A \sqrt{\gamma+r_{s}}} .
$$

Here, $D=3.3953$ and $A=3.2581$ recover Yasuhara's [29] ladder-diagram behavior $D r_{s}^{3 / 2} e^{-A \sqrt{r_{s}}}$ in the low density $\left(r_{s} \rightarrow \infty\right)$ limit, while $\gamma=4.7125$ and $\beta=163.44$ recover Kimball's [30] exact highdensity $\left(r_{s} \rightarrow 0\right)$ limit, $\left(1-0.7317 r_{s}\right) / 2$. An exact generalization of Eq. (15) to the partially spinpolarized case is unknown, but an appropriate one [31] multiplies $g^{\text {unif }}\left(r_{s} ; 0\right)$ by $\left(1-\zeta^{2}\right)$.

To visualize the coupling-constant dependence, it is only necessary to relabel the vertical and horizontal axes of Figure 1 as $\left\langle n_{x c, \lambda}(0)\right\rangle /\langle n\rangle$ and $\lambda\left\langle r_{s}\right\rangle$, respectively. This follows from the invariance of $\left\langle n_{x c, \lambda}(0)\right\rangle /\langle n\rangle$ and $\lambda\left\langle r_{s}\right\rangle$ under the Levy scaling [32] $\lambda \rightarrow \gamma \lambda$ and $\Psi_{\lambda}\left(\mathbf{r}_{1}, \sigma_{1}, \ldots, \mathbf{r}_{N}, \sigma_{N}\right) \rightarrow$ $\gamma^{3 N / 2} \Psi_{\lambda}\left(\gamma \mathbf{r}_{1}, \sigma_{1}, \ldots, \gamma \mathbf{r}_{N}, \sigma_{N}\right)$.

The accuracy of the LSD on-top hole density completes [17] the explanation for the accuracy of LSD or GGA energies in "normal" systems like those shown in Figure 1: The approximate $\left\langle n_{x c, \lambda}(u)\right\rangle$ is accurate for $u \rightarrow 0$. This fact and the sum rule of Eq. (11) constrain the energy integral of Eq. (8) to reasonable values. The on-top hole density provides the "missing link" between real atoms or molecules and the uniform electron gas.

This explanation also works for self-interaction corrected [33] (SIC) LSD, which has [17, 34] the same hole density and cusp in the limit $u \rightarrow 0$ as do LSD and GGA and respects the same sum rule [33].

\section{Normal and Abnormal Systems}

Burke et al. [17] explained the remarkable accuracy of the LSD on-top hole density as follows: For slowly varying spin densities, the system-averaged hole density $\left\langle n_{x c, \lambda}(u)\right\rangle$ has a gradient expression

$$
\begin{aligned}
\left\langle n_{x c, \lambda}(u)\right\rangle= & \left\langle n_{x c, \lambda}^{\mathrm{LSD}}(u)\right\rangle+\frac{1}{N} \int d^{3} r n^{2}(\mathbf{r}) \\
& \times \Gamma\left(\lambda n^{-1 / 3}(\mathbf{r}) ; \zeta(\mathbf{r}) ; u\right) \\
& \times \frac{|\nabla n(\mathbf{r})|^{2}}{n^{8 / 3}(\mathbf{r})}+\cdots .
\end{aligned}
$$

Applied to a slowly or moderately varying density, this expression to second order in $\nabla n$ is realistic for $u \rightarrow 0$ and unrealistic for $u \rightarrow \infty$. $\Gamma$ and the higher gradient coefficients at $u=0$ must vanish in the limits $\lambda \rightarrow 0, \lambda \rightarrow \infty$, and $\zeta \rightarrow 1$ and are apparently otherwise quite small. Moreover, the LSD on-top hole in the approximation of Eqs. (15) and (16) has a Taylor expansion in powers of $\lambda$ or $\left\langle r_{s}\right\rangle$, like the perturbation expansion [35] of the exact on-top hole in a finite system.

Thus, the LSD on-top hole density $\left\langle n_{x c, \lambda}(0)\right\rangle$ is expected to be accurate even when the density is not slowly varying over space-and this accuracy is confirmed in Figure 1 for "normal systems." However, this expectation may be foiled if the correlations are essentially different from those in an electron gas of slowly varying density, i.e., for an "abnormal" system, which displays "static" $[36,37]$ as well as "dynamic" correlations. In a finite system, abnormality typically arises in two ways: (1) If the Kohn-Sham or $\lambda=0$ system has a ground state which is a linear combination of several degenerate Slater determinants (e.g., an atomic triplet state with $S=1$ and $\left.M_{s}=0\right)$, then the LSD on-top hole density can be wrong $[11,18]$ even at the $\lambda=0$ or exchange-only limit. (2) If the Kohn-Sham or $\lambda=0$ system has a nearly degenerate ground state, then the $\lambda$-dependence of $\left\langle n_{x c, \lambda}(0)\right\rangle$ can be very different from its LSD approximation (as illustrated by the stretched $\mathrm{H}_{2}$ example of the fourth section).

From the foregoing, we might expect that LSD and GGA energies are realistic for normal systems, but not for abnormal ones. In fact, self-consistent 
results for these energies are good even for abnormal systems, in all the cases that we know. When the degree of abnormality is mild, so are its effects on the energy. When it is strong, self-consistent LSD and GGA spin densities tend to break symmetry in order to deliver good energies.

As an example of mild abnormality, consider the four-electron ions of nuclear charge $Z \rightarrow \infty$. Because of the near-degeneracy of $(2 s)^{2}$ and $(2 p)^{2}$ singlet configurations [38-40], the ground-state wave function does not reduce to a single determinant when $Z \rightarrow \infty$ and the correlation energy scales like $Z$, in contrast to the "normal" $Z^{0}$ behavior displayed by the two-electron ions.

To better understand the case $N=4$, we performed Hartree-Fock (HF) and minimal multiconfiguration self-consistent field (MCSCF) calculations* for $Z=4,8,12,50$, and 60 , then fitted the results to $Z^{-1}$ expansions. As $Z \rightarrow \infty$, the HF and MCSCF densities $\left[n^{\mathrm{HF}}(\mathbf{r})\right.$ and $\left.n^{\mathrm{MCSCF}}(\mathbf{r})\right]$ each scale like $Z^{3} f(Z r)$ and differ typically by only a few percent. The system-averaged exchange and correlation $(\lambda=1)$ hole densities also separately scale

* A modified version of the COLUMBUS program system [41, 42], as described in [34], was used. The Gaussian basis set for the Be atom was also the same as used in [34]. The exponents of the Gaussians were scaled appropriately, to account for the change in the nuclear charge. like $Z^{3} g(Z u)$ (Figs. 2 and 3), unlike the $Z^{2} g(Z u)$ scaling [43] of the correlation hole for the case $N=2$. When $Z \rightarrow \infty$, we find that the HartreeFock orbital energies have the limits $\epsilon_{2 s} \rightarrow-Z^{2} / 8$, $\epsilon_{2 p} \rightarrow-Z^{2} / 8$, and $\epsilon_{2 p}-\epsilon_{2 s} \rightarrow 0.21 Z$. The energy limits are $E_{x}^{\mathrm{HF}} \rightarrow-0.819 \mathrm{Z}, E_{c}=E^{\mathrm{MCSCF}}-E^{\mathrm{HF}} \rightarrow$ $-0.012 Z$, and $E_{x}\left[n^{\mathrm{MCSCF}}\right]-E_{x}\left[n^{\mathrm{HF}}\right] \rightarrow-0.0005 \mathrm{Z}$, where the last estimate was found using the Perdew-Wang 1991 GGA [5, 6] for the exchange energy $E_{x}[n]$.

From these results, we draw two conclusions about the limit $Z \rightarrow \infty$ : (1) The difference $E_{x}\left[n^{\mathrm{MCSCF}}\right]-E_{x}\left[n^{\mathrm{HF}}\right]$, although nonzero, is less than $5 \%$ of $E_{c}$ and certainly cannot account for the whole linear dependence upon $Z$. Thus, as Perdew et al. [39] suggested, the different Z-scalings of $E_{c}$ for $N=2$ and $N=4$ show that the exact density functional for the correlation energy must be much more sophisticated than any LSD or GGA form. (2) $E_{c}$ is less than $1.5 \%$ of $E_{x}$. Thus, although LSD and GGA fail badly for $E_{c}$ at $N=4$ (predicting a $\ln Z$ or $Z^{0}$ dependence), they work rather well for $E_{x c}$. We propose that $E_{x c}$ in an abnormal system should be regarded as a single indivisible entity. This is also clear for a diatomic molecule like $\mathrm{H}_{2}$ in the limit of large bond length (fourth section), where LSD and GGA describe $E_{x}$ poorly but $E_{x c}$ quite well [36].

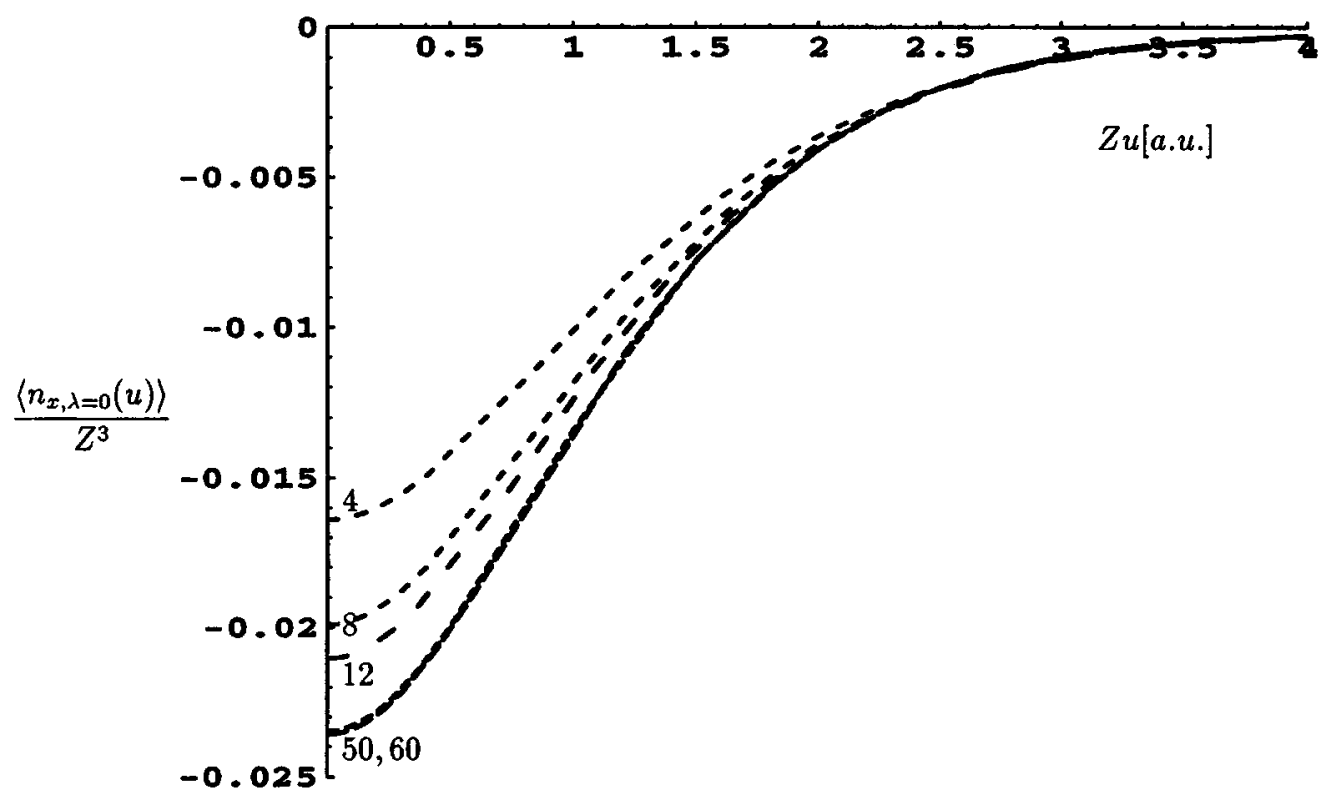

FIGURE 2. Scaled system-averaged exchange hole in four-electron ions with nuclear charge $Z=4,8,12,50$ and 60 , from Hartree-Fock calculations. 


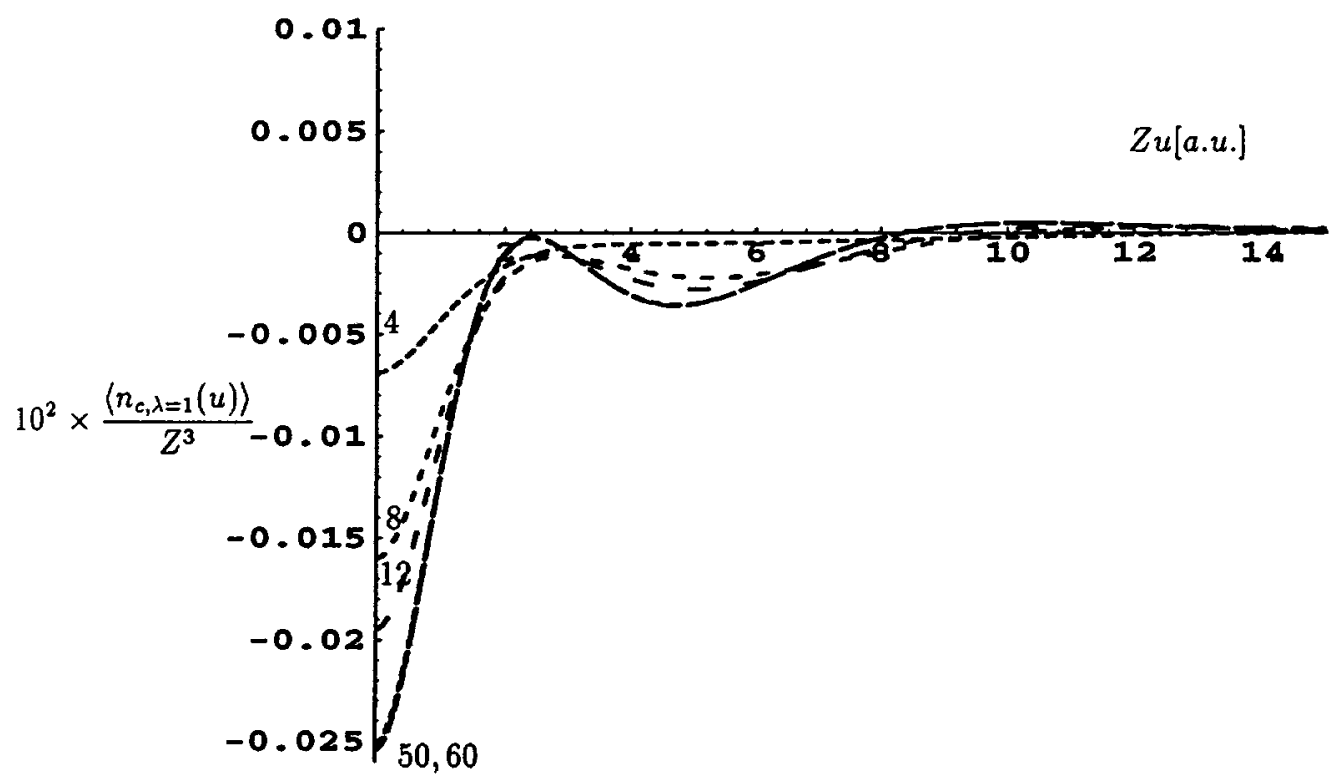

FIGURE 3. Scaled system-averaged correlation hole $(\lambda=1)$ in four-electron ions with nuclear charge $Z=4,8,12,50$, and 60 , from multiconfiguration SCF and Hartree-Fock calculations. Note the difference of scale between Figures 2 and 3.

\section{Alternative Physical Interpretation of Spin Density Functional Theory}

Systems as simple as the molecule $H_{2}$ have much to teach us [15]. The true $(\lambda=1)$ ground state for any bond length is a spin singlet [44] $(S=0)$, with $m(\mathbf{r})=0$ everywhere. At its equilibrium bond length, $H_{2}$ is a "normal" system: Its $\lambda=0$ wave function is a single Slater determinant with one electron in the spin-up bonding orbital and the other in the spin-down bonding orbital. As we stretch the bond length, $\mathrm{H}_{2}$ becomes strongly "abnormal," due to the growing near-degeneracy of the bonding and antibonding orbitals. When the bond length tends to infinity, any positive coupling constant $\lambda$, however small, suffices to stabilize the Heitler-London wave function [which localizes one electron on each atom, making $\left.n_{x c, \lambda}(\mathbf{r}, \mathbf{r})=-n(\mathbf{r})\right]$ with respect to the Kohn-Sham $\lambda=0$ wave function [which contains spurious ionic configurations, like $H^{+} \cdots H^{-}$, making $n_{x}(\mathbf{r}, \mathbf{r})=$ $-n(\mathbf{r}) / 2]$.

The true atomization energy $\mathrm{H}_{2}$ is $4.5 \mathrm{eV}$. If we evaluate [15] the atomization energy from the binding energy curve, using the true spin-unpolarized densities of the separated atoms, we get $6.6 \mathrm{eV}$ in LSD or $6.9 \mathrm{eV}$ in GGA - with significant errors showing that stretched $H_{2}$ is an abnormal system. But, if we use self-consistent LSD or GGA spin densities, we get $4.7 \mathrm{eV}$ in LSD or $4.5 \mathrm{eV}$ in GGA. We get these correct atomization energies from incorrect (broken-symmetry) spin densities which localize a spin-up electron on one atom and a spin-down electron on the other. Similar symmetry breakings occur even at the equilibrium bond length for the singlet open-shell molecules $[6,8]$ $\mathrm{C}_{2}$ and $\mathrm{Cr}_{2}$.

Perdew et al. [15] explained these results via an alternative pair-density interpretation of LSD and GGA calculations, in which the physical predictions are $E, n(\mathbf{r})$, and $P_{\lambda=1}(\mathbf{r}, \mathbf{r})$ instead of $m(\mathbf{r})$. In this interpretation, the self-consistent $n_{\uparrow}(\mathbf{r})$ and $n_{\downarrow}(\mathbf{r})$ are auxiliary quantities like the Kohn-Sham orbitals, which are used to construct the density $n(\mathbf{r})=n_{\uparrow}(\mathbf{r})+n_{\downarrow}(\mathbf{r})$ and the on-top pair density:

$$
P_{\lambda=1}(\mathbf{r}, \mathbf{r})=P_{\lambda=1}^{u n i f}\left(n_{\uparrow}(\mathbf{r}), n_{\downarrow}(\mathbf{r}) ; u=0\right) .
$$

Thus, in stretched $\mathrm{H}_{2}$, the apparent spin polarization of each atom in a self-consistent LSD or GGA calculation is really just a way of making $n_{x c, \lambda}(\mathbf{r}, \mathbf{r})$ $=-n(\mathbf{r})$, and so lowering the negative exchangecorrelation energy of Eq. (8).

Underlying the standard physical interpretation of spin density functional theory is the formally exact theory of Kohn and Sham [1], for which the adiabatic connection $[9,25]$ between interacting 
and noninteracting systems was described in the second section. Underlying the alternative physical interpretation of [15] is a nearly exact variational theory [15], to which LSD and GGA are also valid approximations in the slowly varying limit. In this alternative theory, the variational wave function $\Psi_{\lambda}$ for coupling strength $\lambda$ is constrained to have total density $n(\mathbf{r})=n_{\uparrow}(\mathbf{r})+n_{\downarrow}(\mathbf{r})$ and on-top pair density

$$
P_{\lambda}(\mathbf{r}, \mathbf{r})=P_{\lambda}^{u n i f}\left(n_{\uparrow}(\mathbf{r}), n_{\downarrow}(\mathbf{r}) ; u=0\right),
$$

and minimizes $\hat{T}+\lambda \hat{V}_{e e}$ subject to these constraints. The functions $n_{\uparrow}(\mathbf{r})$ and $n_{\downarrow}(\mathbf{r})$ are varied to minimize the energy, subject to the constraints $n_{\sigma}(\mathbf{r}) \geq 0, \int d^{3} r n_{\sigma}(\mathbf{r})=N_{\sigma}=$ integer and $N_{\uparrow}+N_{\downarrow}$ $=N$. Only at $\lambda=0$ can $n_{\uparrow}(\mathbf{r})$ and $n_{\downarrow}(\mathbf{r})$ be regarded as the spin densities of the wave function $\Psi_{\lambda}$.

Why are the LSD and GGA approximations more faithful to the alternative theory of [15] than to the original theory of Kohn and Sham, in the absence of an external magnetic field? The reason seems to be that the exchange-correlation energy integral of Eq. (8) depends directly upon $P_{\lambda=1}(\mathbf{r}, \mathbf{r})$ or $n_{x c, \lambda}(\mathbf{r}, \mathbf{r})$ and only indirectly upon the spin magnetization $m(\mathbf{r})$. Of course, in the presence of an external magnetic field that couples strongly enough to the spin magnetization, these approximations will be more faithful to the original theory.

\section{Magnetism in Solids}

LSD and GGA successfully predict which metals are ferromagnetic or spin-aligned $(\mathrm{Fe}, \mathrm{Ni}, \mathrm{Co})$ and which are not [45]. Both approximations correctly predict that body-centered cubic (bcc) Fe is magnetic (with the right spin moment per atom) while face-centered cubic (fcc) Fe is not. GGA does slightly better than does the LSD by predicting that the bcc crystal structure has the lower energy [19-21].

We believe that bcc Fe is a "normal" system, in the same way that the nitrogen atom is. A finite cluster or an infinite crystal of this material has a true magnetic ground state with $m(\mathbf{r}) \neq 0$, which is nicely mimicked by the LSD or GGA self-consistent spin densities. The ground state has a high spin $S$ and high $(2 S+1)$-fold degeneracy. The states with $M_{S}= \pm S$, which reduce to single Slater determinants as $\lambda \rightarrow 0$, are not mixed by the Coulomb interaction with states of other $M_{S}$.

It is not even necessary to perform self-consistent spin-polarized calculations to predict whether a crystal will be ferromagnetic. We only need the uniform spin susceptibility $\chi$ of an assumed paramagnetic state, defined through the equation $M / \Omega=\chi B$, where $M=\mu_{e} \int d^{3} r m(\mathbf{r})$ is the magnetic moment of a crystal of volume $\Omega$ in the presence of a uniform weak external magnetic field $B$. The associated total-energy change is $-M B+M^{2} / 2 \chi \Omega$; the energy of spontaneous magnetization $\left(M^{2} / 2 \chi \Omega\right)$ has the same sign as $\chi$. Vosko and Perdew [46] derived a variational principle for $\chi^{-1}$ in which the normalized trial function which delivers the minimum and true $\chi^{-1}$ is $\mu_{e} m(\mathbf{r}) / M$. Approximating this trial function by its noninteracting or $\lambda=0$ limit

$$
\gamma(\mathbf{r})=\sum_{i} \delta\left(\boldsymbol{\epsilon}_{F}-\boldsymbol{\epsilon}_{i}\right)\left|\psi_{i}(\mathbf{r})\right|^{2} / \sum_{i} \delta\left(\boldsymbol{\epsilon}_{F}-\boldsymbol{\epsilon}_{i}\right),
$$

the normalized Fermi-level density of the KohnSham system, they proved that

$$
\chi^{-1} \leq \chi_{\mathrm{VP}}^{-1}
$$

where

$$
\chi_{\mathrm{VP}}=\frac{\mu_{e}^{2} N\left(\boldsymbol{\epsilon}_{F}\right)}{1-N\left(\boldsymbol{\epsilon}_{F}\right) I} .
$$

Here, $N\left(\epsilon_{F}\right)=\Omega^{-1} \sum_{i} \delta\left(\epsilon_{F}-\epsilon_{i}\right)$ is the noninteracting Fermi-level density of states, and

$$
I=-\left.\Omega \int d^{3} r \int d^{3} r^{\prime} \gamma(\mathbf{r}) \frac{\delta^{2} E_{x c}[n, m]}{\delta m(\mathbf{r}) \delta m\left(\mathbf{r}^{\prime}\right)}\right|_{m=0} \gamma\left(\mathbf{r}^{\prime}\right) .
$$

The Stoner parameter I can be evaluated from Eq. (23) within LSD, including the effects of exchange (which favors magnetism) and correlation (which opposes it). $N\left(\epsilon_{F}\right)$ and $I$ are both positive, but $\chi_{\mathrm{VP}}$ and, hence, $\chi$ will be negative when $N\left(\epsilon_{F}\right) I>1$.

Starting from a spin-unpolarized band-structure calculation, Janak [45] evaluated $\chi_{\mathrm{VP}}$ within LSD. When $\chi_{\mathrm{VP}}$ is positive (as it is for most metals), it usually provides a realistic estimate for $\chi$. When $\chi_{\mathrm{VP}}$ is negative (as for $\mathrm{Fe}$ and $\mathrm{Ni}$ ), then $\chi$ is also negative by Eq. (21) and the spin-unpolarized state is unstable. In the case of $\mathrm{Co}, \chi_{\mathrm{VP}}$ is positive and very large, a result which suggests (but does not establish) the ferromagnetism of this metal. 
Gunnarsson [24] independently derived $\chi_{\mathrm{VP}}$ of Eq. (22) as an approximation (not as a bound) and introduced a finite temperature into the Kohn-Sham orbital occupation numbers in order to estimate the transition temperature $T(V P)$ above which $\chi_{\mathrm{VP}}$ is positive for Fe. This temperature, shown in Table I, is considerably higher than the Curie temperature at which the local spin moments on the atomic sites disorder and fluctuate freely [making $m(\mathbf{r})=0$ ]. The size and sign of this discrepancy pose problems for the standard interpretation of LSD spin density functional theory. Table I also shows that Gunnarsson's transition temperature is much closer to the Stoner temperature, at which the local moments vanish, than to the Curie temperature at which $m(\mathbf{r})$ vanishes.

The local moment on a site persists for a time long compared to that for an electron to move across the site [49]. By treating the fast degrees of freedom via LSD and the slow degrees of freedom by statistical mechanics, Pindor et al. [47, 48] calculated the magnitude of the local moment and the Stoner temperature at which it vanishes (Table I).

We can perhaps understand Gunnarsson's results in terms of our on-top pair-density interpretation. Thermal fluctuation of the local moments does not greatly change the on-top pair density, which is associated with the fast electronic degrees of freedom. But the disappearance of these moments at the Stoner temperature will certainly be reflected in the on-top pair density.

A full resolution of this issue may require improved calculations. Gunnarsson's pioneering work, now 20 years old, is not self-consistent and does not use the best current electron-gas input. His zero-temperature $\chi_{\mathrm{VP}}$ for Co differs in sign from that of Janak [45], and his transition tempera-

\section{TABLE 1}

\section{Transition temperatures (in K) for solid} ferromagnetic iron.

\begin{tabular}{|c|c|c|}
\hline$T(V P)$ & $T$ (Curie) & $T$ (Stoner) \\
\hline $4400-6200^{a}$ & $1040,^{a} 1250^{b}$ & $5000-5500^{c}$ \\
\hline \multicolumn{3}{|c|}{$\begin{array}{l}T(V P) \text { is the temperature above which } \chi_{V P} \text { of Eq. (22) is } \\
\text { positive. } T(\text { Curie) is the observed [24] or theoretical [47] } \\
\text { Curie temperature at which the local spin moments disorder } \\
\text { and fluctuate freely. } T \text { (Stoner) is the theoretical [48] Stoner } \\
\text { temperature, at which the local moments disappear. (Iron } \\
\text { melts at } 1810 \mathrm{~K} .) \\
\text { a }[24] \text {. } \\
\text { b [47]. }\end{array}$} \\
\hline
\end{tabular}

ture for Ni $(2900 \mathrm{~K})$ differs from that of Liu et al. [50] (1780 K).

We have argued that ferromagnetic iron is a normal system at zero temperature. In such a system, we can trust LSD or GGA predictions for the ground-state spin magnetization $m(\mathbf{r})$ and, hence, for the zero-temperature local moment. We suspect, however, that clusters of antiferromagnetic solids (with antialigned local moments) [22, 23] like $\mathrm{FeO}$ or $\mathrm{Cr}$ are abnormal, because their LSD or GGA ground-state spin densities break a symmetry. To see this, consider a finite cluster of antiferromagnetic material in a singlet ground state with spin magnetization $m(\mathbf{r})=0$. LSD and GGA will predict $n_{\uparrow}(\mathbf{r})-n_{\downarrow}(\mathbf{r}) \neq 0$ a symmetry breaking not allowed in the exact theory except in the thermodynamic or infinite-volume limit [51]. In other words, ground-state antiferromagnetism in a finite cluster is really a long-range correlation somewhat like that between the two electrons in stretched $\mathrm{H}_{2}$. If antiferromagnets are abnormal systems, then LSD or GGA predictions for their local spin moments may not be so reliable as those for groundstate ferromagnets like Fe. A necessary condition for a reliable prediction is that the time over which a local moment fluctuates must be much longer than other electronic time scales.

\section{ACKNOWLEDGMENTS}

This work is dedicated to the memory of Sy Vosko and was supported by the National Science Foundation under Grant No. DMR95-21353 and by the Deutsche Forschungsgemeinschaft.

\section{References}

1. W. Kohn and L. J. Sham, Phys. Rev. 140, A 1133 (1965).

2. R. M. Dreizler and E. K. U. Gross, Density Functional Theory (Springer-Verlag, Berlin, 1990).

3. R. G. Parr and W. Yang, Density Functional Theory of Atoms and Molecules (Oxford, New York, 1989).

4. R. O. Jones and O. Gunnarsson, Rev. Mod. Phys. 61, 689 (1989).

5. J. P. Perdew, in Electronic Structure of Solids '91, P. Ziesche and H. Eschrig, Eds. (Akademie Verlag, Berlin, 1991); J. P. Perdew, K. Burke, and Y. Wang, unpublished.

6. J. P. Perdew, J. A. Chevary, S. H. Vosko, K. A. Jackson, M. R. Pederson, D. J. Singh, and C. Fiolhais, Phys. Rev. B 46, 6671 (1992); Ibid. 48, 4978 (1993) (E).

7. O. Gunnarsson, B. I. Lundqvist, and J. W. Wilkins, Phys. Rev. B 10, 1319 (1974) 
8. B. Delley, A. J. Freeman, and D. E. Ellis, Phys. Rev. Lett. 50, 488 (1983); B. I. Dunlap, Phys. Rev. A 27, 2217 (1983); B. I. Dunlap, Phys. Rev. A 27, 2217 (1983).

9. O. Gunnarsson and B. I. Lundqvist, Phys. Rev. B 13, 4274 (1976).

10. O. Gunnarsson, M. Jonson, and B. I. Lundqvist, Phys. Rev. B 20, 3136 (1979).

11. T. Ziegler, A. Rauk, and E. J. Baerends, Theor. Chim. Acta 43, 261 (1977).

12. J. Harris, Phys. Rev. A 29, 1648 (1984).

13. J. P. Perew, Phys. Rev. Lett. 55, 1665 (1985); Ibid. 55, 2370 (1985) (E).

14. K. Burke, J. P. Perdew, and D. C. Langreth, Phys. Rev. Lett. 73, 1283 (1994).

15. J. P. Perdew, A. Savin, and K. Burke, Phys. Rev. A 51, 4531 (1995).

16. K. Burke and J. P. Perdew, Mod. Phys. Lett. B 9, 829 (1995).

17. K. Burke, J. P. Perdew, and M. Ernzerhof, unpublished.

18. A. D. Becke, A. Savin, and H. Stoll, Theor. Chim. Acta 91, 147 (1995).

19. C. S. Wang, B. M. Klein, and H. Krakauer, Phys. Rev. Lett. 54, 1852 (1985).

20. P. Bagno, O. Jepsen, and O. Gunnarsson, Phys. Rev. B 40, 1997 (1989).

21. D. J. Singh, W. E. Pickett, and H. Krakauer, Phys. Rev. B 43, 11628 (1991).

22. P. Dufek, P. Blaha, V. Sliwko, and K. Schwarz, Phys. Rev. B 4910170 (1994).

23. D. J. Singh and J. Ashkenazi, Phys. Rev. B 46, 11570 (1992).

24. O. Gunnarsson, J. Phys. F 6, 587 (1976).

25. D. C. Langreth and J. P. Perdew, Solid State Commun. 17, 1425 (1975); Phys. Rev. B 15, 2884 (1977).

26. M. Levy and J. P. Perdew, Phys. Rev. A 32, 2010 (1985).

27. D. C. Langreth and J. P. Perdew, Solid State Commun. 31, 567 (1979); Phys. Rev. B 21, 5469 (1980).

28. M. Taut, Phys. Rev. A 48, 3561 (1993).

29. H. Yasuhara, Solid State Commun. 11, 1481 (1972).
30. J. C. Kimball, Phys. Rev. B 14, 2371 (1976); D. J. W. Geldart, Can. J. Phys. 45, 3139 (1967).

31. J. P. Perdew and Y. Wang, Phys. Rev. B 46, 12947 (1992).

32. M. Levy, Phys. Rev. A 43, 4637 (1991).

33. J. P. Perdew and A. Zunger, Phys. Rev. B 23, 5048 (1981).

34. M. Ernzerhof, K. Burke, and J. P. Perdew, submitted.

35. A. Görling and M. Levy, Phys. Rev. B 47, 13105 (1993).

36. V. Tschinke and T. Ziegler, J. Chem. Phys. 93, 8051 (1990).

37. R. Neumann, R. H. Nobes, and N. C. Handy, unpublished.

38. J. Linderberg and H. Shull, J. Mol. Spectrosc. 5, 1 (1960).

39. J. P. Perdew, E. R. McMullen, and A. Zunger, Phys. Rev. A 23, 2785 (1981).

40. T. Zhu, C. Lee, and W. Yang, J. Chem. Phys. 98, 4814 (1993).

41. R. Shepard, I. Shavitt, R. M. Pitzer, D. C. Comeau, M. Pepper, H. Lischka, P. G. Szalay, R. Ahlrichs, F. B. Brown, and J.-G. Zhoa, Int. J. Quantum Chem. 142, 22 (1988).

42. R. Shepard, H. Lischka, P. G. Szalay, T. Kovar, and M. Ernzerhof, J. Chem. Phys. 96, 2085 (1992).

43. F. Arias de Saavedra, E. Buendiá, and F. J. Gálvez, J. Phys. B 27, 4433 (1994), see Fig. 1.

44. N. W. Ashcroft and N. D. Mermin, Solid State Physics (Holt, Rinehart, and Winston, New York, 1976), problem 2 of Chap. 2.

45. J. F. Janak, Phys. Rev. B 16, 255 (1977).

46. S. H. Vosko and J. P. Perdew, Can. J. Phys. 53, 1385 (1975).

47. B. L. Gyorffy, A. J. Pindor, J. B. Staunton, G. M. Stocks, and H. Winter, J. Phys. F 15, 1337 (1985).

48. A. J. Pindor, J. Staunton, G. M. Stocks, and H. Winter, J. Phys. F 13, 979 (1983).

49. B. L. Gyorffy, J. B. Staunton, and G. M. Stocks, in Density Functional Theory, E. K. U. Gross and R. M. Dreizler, Eds. (Plenum, New York, 1995).

50. K. L. Liu, A. H. MacDonald, and S. H. Vosko, Inst. Phys. Conf. Ser. 39, 557 (1978).

51. T. A. Kaplan, P. Horsch, and W. von der Linden, J. Phys. Soc. Jpn. 58, 3894 (1989). 